\title{
Imbibition in porous media: correlations of displacement events with pore-throat geometry and the identification of a new type of pore snap-off
}

\author{
Kamaljit Singh $^{1,2, *}$, Tom Bultreys ${ }^{3,4}$, Ali Q. Raeini ${ }^{3}$, Mosayeb Shams $^{3}$, and Martin J. Blunt ${ }^{2}$ \\ ${ }^{1}$ Institute of GeoEnergy Engineering, Heriot-Watt University, EH14 4AS Edinburgh, U.K. \\ ${ }^{2}$ Qatar Carbonates and Carbon Storage Research Centre, Department of Earth Science and Engineering, Imperial College \\ London, SW7 2AZ London, U.K. \\ ${ }^{3}$ Department of Earth Science and Engineering, Imperial College London, SW7 2AZ London, U.K. \\ ${ }^{4}$ Department of Geology, Ghent University, 9000 Ghent, Belgium \\ ${ }^{*}$ Corresponding author: Kamaljit Singh, k.singh@hw.ac.uk
}

The displacement of a non-wetting fluid by a wetting fluid in porous media, called imbibition, is important in many natural and industrial processes. During imbibition, the wetting fluid invades the pore space through a series of competitions between piston-like displacement, film and corner flow, snapoff, pore bypassing and trapping. Our understanding of these fundamental pore-scale displacement processes in natural three-dimensional porous media with complex pore geometries is still incomplete. Here, we use time-resolved three-dimensional synchrotron X-ray micro-tomography to visualize and quantify these processes in a water-wet carbonate rock sample. We utilize a pore-throat network extracted from the segmented pore space and superimpose it on experimental three-dimensional timeseries datasets to obtain properties of pores and throats involved in 183 displacement events. Our findings suggest that the occurrence of snap-off or $I_{n}$ pore-filling events strongly correlates with the aspect ratio (the ratio of pore radius to throat radius involved in the displacement event), and weakly with the throat shape factor and other pore-throat parameters. In addition, we have found a new type of snap-off event that occurs in pores rather than in throats, which is related to the pinning of fluidfluid interfaces at rough surfaces resulting in a contact angle more than $90^{\circ}$ during displacement. This type of pore snap-off is independent of the aspect ratio and leads to the formation of a non-wetting fluid ganglion occupying a fraction of a single pore body. By incorporating these new events and the correlation of displacement events with pore-throat geometry, existing models can be improved and validated.

Keywords: Imbibition, snap-off, pore-filling, porous media, 3D imaging

\section{Introduction}

Multiphase flow, including drainage and imbibition, in porous media occur, for instance, during water infiltration in soils, geo-sequestration of supercritical $\mathrm{CO}_{2}$ in deep saline aquifers or depleted oil and gas fields, subsurface non-aqueous phase liquid contaminant transport, and oil recovery from reservoir rocks $(1,2)$. At low injection rates, typical in earth science applications, capillary forces tend to dominate drainage and imbibition, which consist of a sequence of several types of fluid displacement events (3-8). The displacement of a wetting fluid by a non-wetting fluid from porous media is called drainage, whereas the displacement of a non-wetting fluid by a wetting fluid is called imbibition. Drainage is controlled by the narrowest regions, called throats, between the adjoining widest regions, called pores, and consists of Haines jumps (in which fluid-fluid interface makes a sudden and fast jump through the throat to the adjacent pores, halting at the next throat which has a higher threshold pressure) and Roof-snap-off events (3,913).

On the other hand, imbibition is controlled by both pores and throats (14). During secondary imbibition, when the wetting fluid is initially present in small pores and throats as well as in pore corners before imbibition, the two most important processes that compete are pore-filling and snap-off $(15-17)$. These processes strongly influence the trapping of the non-wetting phase which is reflected in oil recovery, $\mathrm{CO}_{2}$ storage and remediation efficiency of contaminated land. The pioneering work of Lenormand and co-workers $(4,16)$, using a twodimensional micro-model consisting of a square lattice with a co-ordination number of four, provided 
a detailed analysis of threshold capillary pressure required for pore-filling events under different fluid configurations, called $I_{n}$ events. Figure 1 a-c show schematics of three different fluid configurations. In $I_{1}$ displacement events, only one of the connecting throats is initially filled with a non-wetting fluid (Figure 1a). Similarly, in $I_{2}$ and $I_{3}$ events, two and three connecting throats are initially filled with a non-wetting fluid respectively (Figure 1 b-c). During waterflooding, pore-filling is more favorable for $I_{1}$ compared to $I_{2}$ for the same pore size, as $I_{1}$ can happen at higher capillary pressure that can be estimated using simple geometrical calculations and the Young-Laplace equation. A lower curvature and capillary pressure is needed to displace the non-wetting phase in $I_{2}$ (see the dashed oil-brine interfaces in Figure $1 \mathrm{a}-\mathrm{b})$. Similarly, $I_{3}$ is the least favorable among these three displacement events.

Apart from these $I_{n}$ pore-filling mechanisms, a nonwetting fluid can be snapped-off and expelled from a throat when wetting layers in throat corners swell and eventually touch, leading to an unstable interface $(1,3,18)$. Figure $1 \mathrm{~d}$-g show a schematic of such an event for an $I_{1}$ fluid configuration. In this case, the non-wetting fluid in the pore space can either be expelled in a piston-like displacement (Figure 1e), or it can be trapped due to wetting layer swelling in the neighbouring throat leading to the snap-off of the non-wetting phase (Figure 1f-g). A snap-off can also occur without trapping, as shown in (Figure 1h-i). In this case, after a snap-off event, fluid configurations are reduced in order from $I_{n}$ to $I_{n-1}$ (e.g., $I_{2}$ to $I_{1}$ in Figure 1 h-i), which can lead to the displacement of the non-wetting phase by a filling process which was not favorable previously.

The prevalence of snap-off is a function of porethroat geometry and wettability (described by fluidfluid-solid contact angle). Among various geometrical parameters, aspect ratio (the ratio of pore radius to throat radius), angularity or shape and coordination number (the number of throats connected to a pore in consideration) have been investigated in previous two-dimensional and numerical simulation studies $(1,14,19-21)$. In a two-dimensional system, it has been reported that the minimum (threshold) value of aspect ratio for which snap-off events occur increases from 1.5 to 1.75 for an increase in contact angle from $0^{\circ}$ to $55^{\circ}(14,20)$. The threshold aspect ratio becomes infinite for contact angles larger than $70^{\circ}$, and snap-off cannot occur in throats. In general, snap-off is theorized to be the preferred filling mechanism when the aspect ratio is larger than 2 and the contact angle is close to $0^{\circ}$ (for strongly wetting or hydrophilic conditions) (1).
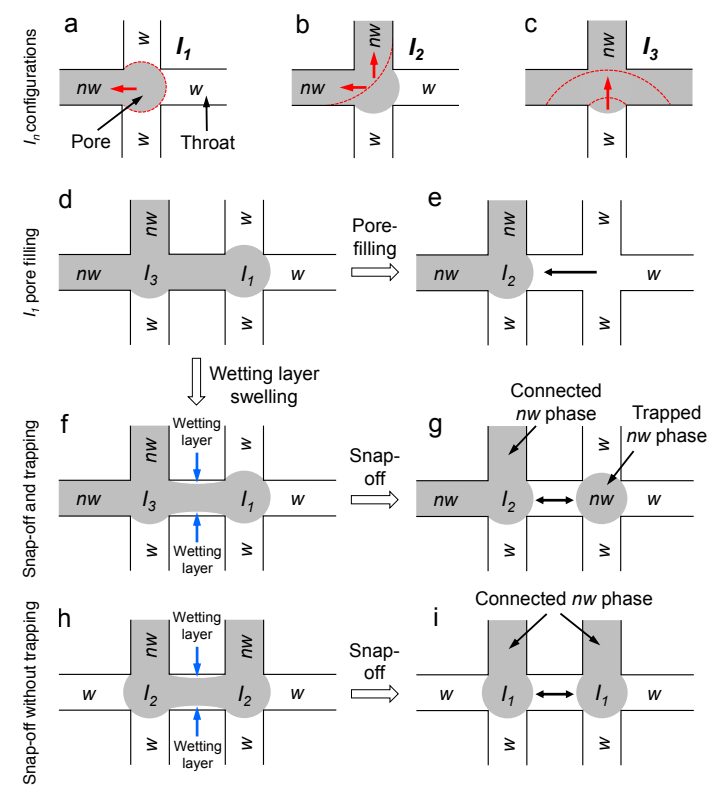

Figure 1: $I_{n}$ pore-filling and snap-off with and without trapping. (a-c) $I_{n}$ pore-filling description in a square lattice with a co-ordination number of four (after Lenormand and Zarcone (12)). A displacement event is called $I_{1}$ if one of the throats is initially filled with a non-wetting fluid (a). Similarly, $I_{2}$ and $I_{3}$ pore-filling events are defined when two and three throats are initially filled with the non-wetting fluid respectively ( $\mathrm{b}$ and $\mathrm{c}$ respectively). $w$ and $n w$ refer to wetting and non-wetting fluids respectively. Red arrows show the direction of fluid displacement. (d-g) An $I_{1}$ configuration experiencing either pore-filling ( $\mathrm{d}$ and $\mathrm{e}$ ) or wetting layer swelling (f) leading to snap-off and trapping (g). (h-i) Snap-off without trapping. Wetting layer swelling between two connected pores with an $I_{2}$ configuration (h) leading to snap-off (i). In this case, the non-wetting fluid after snap-off is still connected.

Secondly, snap-off is favorable for more angular throats in which wetting layers can reside and swell with time (with decreasing capillary pressure or increasing water pressure). Angularity is often characterized by calculating a "shape factor", traditionally defined as $G=A / P^{2}$, where $A$ is the crosssectional area and $P$ is the perimeter of a pore or throat (22). Lastly, snap-off is less likely to occur in a well-connected pore space (described by the coordination number) (1). Most of the available studies on snap-off and pore-filling have focused on two-dimensional systems; however, we still lack a clear understanding of these processes in natural three-dimensional porous media as these have relatively complex pore geometries compared to previously used model systems.

With recent advances in computational power and pore-scale imaging using synchrotron X-ray microtomography, the fluid displacement in porous media can be observed directly in three dimensions at the micrometer scale. This technique has been used 
to study the dynamics of burst instabilities after Haines jumps $(10,11,23)$ and Roof snap-off (3, 13) during drainage. In general, the drainage process is fairly well understood; however, secondary imbibition has not been studied in such detail. Only a handful studies have investigated the threedimensional dynamics of imbibition processes, e.g., (3, 18, 23-28). In terms of morphological features, Herring et al. (29) and Andersson et al. (30) have reported a strong correlation of residual saturation of the non-wetting phase with morphological and persistent homology-based aspect ratios and a weaker correlation with the traditional pore-throat aspect ratio. On the other hand, Tanino and Blunt (31) reported a good correlation between residual saturation and the traditional aspect ratio. In most of the previous studies, an average aspect ratio (ratio of the pore radius to the mean of all bounding throat radii) was used, which does not directly reflect local trapping and pore-filling events. Moreover, a pore-by-pore analysis of various displacement events has not been explored yet, possibly due to lack of time-resolved tomographic data.

In this study, we have used synchrotron X-ray micro-tomography to capture pore-to-pore displacement during capillary-controlled secondary imbibition in a water-wet Ketton limestone sample. We have analyzed and correlated various pore-scale displacement and trapping events to pore and throat geometrical parameters extracted from the segmented pore space (32). We also report a new type of pore snap-off mechanism leading to the trapping of the non-wetting fluid in a fraction of a single pore body. Note that this work is purely based on the analysis of experimentally observed displacement events. We have used pore-throat parameters obtained from a pore-network extraction code; however, the pore-network flow model is not used in this study. A comparison of fluid displacement observed in this experiment to quasi-static pore network flow model simulations are reported elsewhere (33, 34).

\section{Materials and Methods}

\section{Materials}

The experiment was conducted on a $3.8 \mathrm{~mm}$ diameter and $10 \mathrm{~mm}$ long water-wet Ketton limestone rock sample from the Ketton quarry, Rutland, UK, which contains $99.1 \%$ calcite with the remaining fraction being quartz (35). These samples were cleaned with methanol using Soxhlet extraction apparatus for 24 hours, followed by drying in a vacuum oven at $100^{\circ} \mathrm{C}$ for 24 hours. A solution of potassium iodide (KI) salt (puriss, $99.5 \%$, Sigma-Aldrich, UK) in deionized water with a salin- ity of $1.8 \mathrm{~mol} / \mathrm{L}$ was used as the aqueous phase, which provided an effective X-ray contrast between brine (wetting phase) and oil (non-wetting phase). Decane (ReagentPlus, $\geq 99 \%$, Sigma-Aldrich, UK) was used as the oil phase.

\section{Experimental protocol}

The rock sample was placed on a water-wet porous plate in a Viton sleeve. The assembly was loaded in a Hassler-type flow cell made of carbon fiber that is nearly transparent to X-rays. The end pieces that were fitted to either sides of the sleeve were connected to high-pressure pumps (the details of experimental methodology are presented in Singh et al. $(18,36))$. First, the sample was saturated with brine. The system was then pressurized to $10 \mathrm{MPa}$ with a confining pressure of 11.2 MPa. The oil was then injected from the top at a constant pressure drop of $50 \mathrm{kPa}$ to start drainage. Once there was no further displacement of brine from the sample, the flow was reversed by injecting brine from the base of the sample at a constant pressure drop of $22 \mathrm{kPa}$, to start secondary imbibition. The capillary number $N_{c}=v \mu / \sigma$ (where $v$ is the Darcy velocity of the invading fluid, $\mu$ is the viscosity of the invading fluid, and $\sigma$ is the brine-oil interfacial tension) during brine flooding was $1.26 \times 10^{-9}$, representing a capillary-dominated flow regime. The sample was imaged continuously during drainage and secondary imbibition using synchrotron X-ray microtomography at the Diamond Light Source, with a voxel size of $3.28 \mu \mathrm{m}$ and a time-step between consecutive images of $38 \mathrm{~s}$. These tomographic datasets have been uploaded on a public repository (BGS), the details of which are provided in Singh et al. $(36)$.

\section{Image processing}

The reconstructed tomographic images were filtered using an edge preserving non-local mean filter (37). The dry filtered image (without fluids) was segmented into two phases (pores and grains) using a seeded watershed algorithm. We then obtained parameters of pores and throats from the isolated pore space using a pore-throat network approach (32). This method uses a watershed algorithm on the distance map of the segmented pore space image to identify throats (local constrictions) as boundary surfaces between adjacent pores.

The time-series data containing oil and brine phases were subtracted from the initial image of the rock saturated with brine, to isolate the oil phase, which was then segmented using simple thresholding. The segmented oil was applied as a mask on the pore- 
throat network to identify the pores and throats that were occupied by oil and brine. By repeating this procedure for each time step (for 285 tomograms), we obtained information on the characteristics of pores and throats involved in each snap-off and pore-filling event. Note that this analysis on time-resolved images was conducted manually for each time step for quality control. We discarded the events that occurred at the periphery of the sample and at the top and the base of the sample, as the pore-network extraction at these boundaries can result in inaccurate assignment of pore and throat radii.

\section{Statistical analysis}

The correlation of two variables was obtained by calculating Pearson's correlation coefficient (Pearson's $r$ ).

$$
r=\frac{\sum_{i=1}^{n}\left(x_{i}-\bar{x}\right)\left(y_{i}-\bar{y}\right)}{\sqrt{\sum_{i=1}^{n}\left(x_{i}-\bar{x}\right)^{2}} \sqrt{\sum_{i=1}^{n}\left(y_{i}-\bar{y}\right)^{2}}}
$$

where $\bar{x}$ and $\bar{y}$ are the mean values of variables $x$ and $y$ respectively. $x_{i}$ and $y_{i}$ are individual data points of variables $x$ and $y$ respectively. The values of Pearson's $r$ can range from -1 to +1 , where values close to -1 and +1 indicate strong correlations between two variables, whereas a value of 0 shows no correlation.

Secondly, we have calculated $p$-value, which is a probability test that describes whether the null hypothesis is true or there is any relationship between two variables. The $p$-value is based on the $t$-test, described as:

$$
t=\frac{r \sqrt{n-2}}{\sqrt{1-r^{2}}}
$$

where $r$ is Pearson's correlation coefficient, and $n$ is the number of data points. The $p$-value can be determined from $t$-test and the degrees of freedom $(f=n-2)$ using a $t$-distribution table. A $p$-value close to zero indicates that there is a strong relationship between two variables, whereas a high value close to 1 indicates either the lack of correlation (null hypothesis) or that the data are insufficient to prove a correlation.

\section{Results and Discussion}

A sequence of pore-filling and snap-off processes during secondary imbibition is shown in Singh (38) (or doi.org/10.6084/m9.figshare.4235381.v1). During brine injection, the brine-oil interface moves from pore to pore in a piston-like displacement without oil trapping, until a pressure disequilibrium condition is reached, at which the brine layer in the throat corners (ahead of an oil-filled pore) starts to grow. The brine layer continues to grow until the interface is no longer stable, and results in snap-off of the interface and trapping of oil. In our previous study (18), we investigated the mechanism of snap-off by calculating local capillary pressures (obtained from liquid-liquid curvatures) and timescales of brine layer swelling. In this study, we have extended our analysis to obtain conditions that are favorable for pore-filling and snap-off based on porethroat geometry.

\section{Pore-throat geometry and fluid dis- placement events: A localized subset analysis}

Here in this section, we provide a pore-scale displacement analysis on a small subset (an example case as shown in Figure 2) of the scanned image, and then extend this analysis to the complete image in the next section. Figure 2a shows a network of pores (spheres) and throats (tubes) in the subset. The pores are the largest inscribed spheres in the separated pore space, which are colored according to size. Throats are the narrowest constriction along the medial axis connecting two adjacent pores. An illustration of a throat between two pores in two dimensions is shown in Figure $2 \mathrm{~b}$.

The oil phase from the time-resolved segmented data (at $t=25 \mathrm{~min}, 58 \mathrm{~s}$ ) is superimposed on the pore-throat network as shown in Figure 2c. With continuous brine flooding, the interface jumped to adjacent pores (Figure 2d), which is indicated by yellow arrows for pore-filling event $A$ and $B$. By subtracting consecutive images and superimposing them on the pore-throat network, we obtained information on the characteristics of pore and throats involved in these pore-filling events. Similarly, we performed this analysis on the complete image sequence in this subset containing nine events (the video is available in (39) or doi.org/10.6084/m9.figshare.9034367.v1). Events $A$ to $I$ (Figure $2 \mathrm{c}-\mathrm{i}$ ) are presented in a sequence according to the time they occurred. In this subset, eight pore-filling events $(A, B, C, D, E, G, H$ and $I$ ) and one snap-off event $(F)$ can be observed. For each event, we calculated the aspect ratio (the ratio of pore radius to throat radius involved in the event). For example, if the interface jumped from Pore 1 to Pore 2 in Figure 2b, the aspect ratio of the event is calculated as the ratio of the radius of 


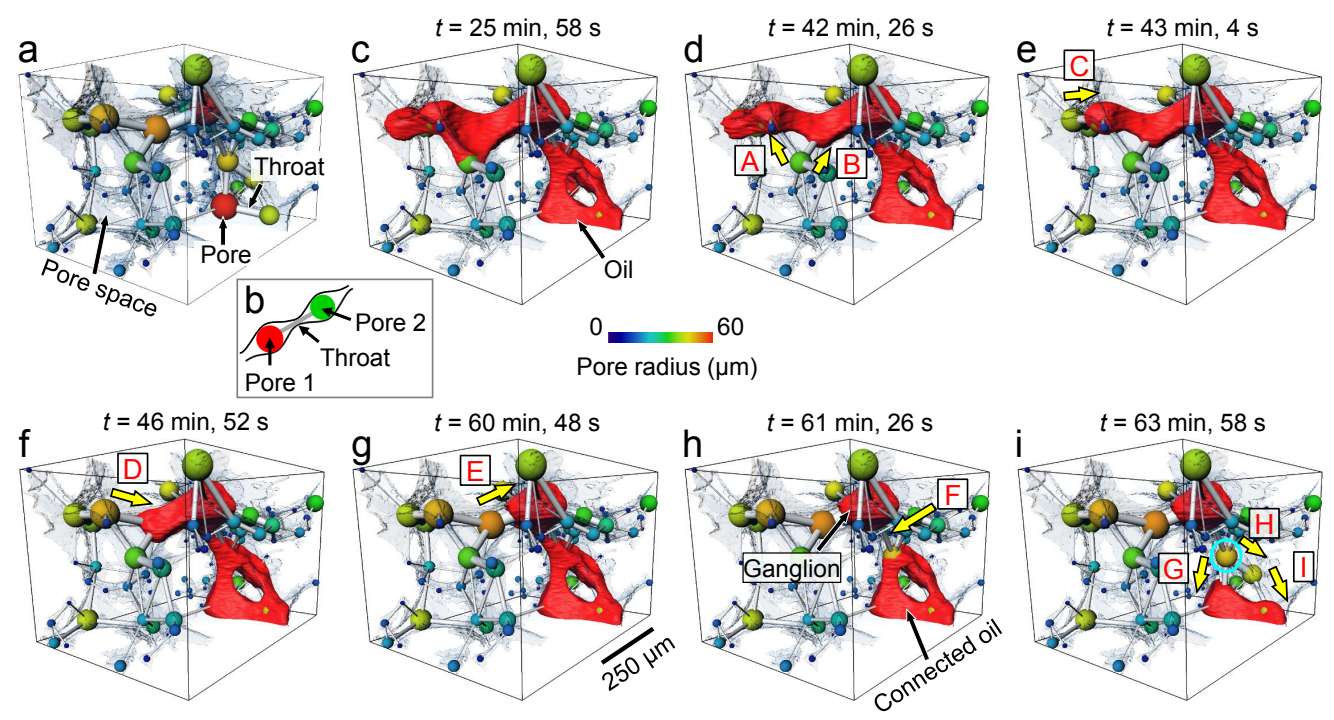

Figure 2: Pore-filling and snap-off events during secondary imbibition in a carbonate rock. (a) A pore-throat network generated from the pore space of the segmented image was used to analyze the characteristics of filling events observed in the experiment. Different colors represent pores according to their sizes. (b) A sketch of pore and throat extraction. The pores are represented as maximum radius circles (spheres in 3D) that fit into the pores space, such as Pore 1 and Pore 2. Throats are represented as tubes connecting two pores. The tube has the same radius as the constriction separating the pores. (c) The oil phase from time-resolved tomography (at $t=25 \mathrm{~min}, 58 \mathrm{~s}$ ) was superimposed on the pore-throat network shown in (a). (c-i) Various time steps showing different displacement events (pore-filling and snap-off) during secondary imbibition. Here, brine and rock are shown transparent and semi-transparent respectively for effective visualization.

Pore 1 and the radius of the throat connecting the two pores. We also calculated shape factors of pores and throats involved in each event. Here, the shape factor is defined as $G=R^{2} /(4 A)$ where $R$ is the radius of the inscribed sphere, and $A$ is the crosssectional area of the pore or throat $(32,40)$. Note that this description of the shape factor is different from the traditional definition.

From the analysis, we find a significant difference in the aspect ratios and throat shape factors for pore-filling events compared to those for snap-off events. The aspect ratio for pore-filling events is in the range of 1.2 to 1.6 , except for the event $I$ with a value of 2.1. An aspect ratio less than 1.6 appears to be favorable for pore-filling. Note that the event $H$ and event $I$ occurred during the same time step after the snap-off event $F$. The interfacial jump after event $F$ could create sufficient pressure fluctuations in the adjacent pores, which could lead to the pore-filling event for an unfavorable aspect ratio of 2.1 in event $I$. The pore-scale fluctuations and inertial effects after a snap-off event have also been observed in a recent study by Rücker and coworkers (20). On the other hand, the aspect ratio for the snap-off event $F$ is 3 , which is significantly larger than that for pore-filling events, therefore, resulting in unfavorable conditions for pore-filling.

The shape factor of throats involved in pore-filling events is in the range of 0.029 to 0.049 , whereas for the snap-off event $F$, the value is 0.026 . The lower value of throat shape factor is favorable for snap-off, since this represents throats with a large surface area and smaller corner angles, which are likely to have more corners in which wetting layers can reside and swell with time (with decreasing capillary pressure or increasing water pressure) leading to snap-off. Although this example subset shows that a lower value of throat shape factor is favorable for snap-off, our analysis on the whole image of the complete time-series datasets (reported in the forthcoming section) shows that the throat shape factor is poorly correlated with displacement events.

\section{Correlation analysis of event types (snap-off and $\boldsymbol{I}_{n}$ pore-filling) and pore- throat geometry: Complete tomo- graphic datasets}

We now extend the subset analysis in Figure 2 to the complete scanned image of the whole time-series (285 tomograms). Table 1 shows the frequency of different snap-off and $I_{n}$ events observed during brine injection. The majority of fluid displacements were $I_{1}$ and $I_{2}$ events, which occurred more frequently than snap-off. Only three $I_{3}$ events were observed in the data analyzed. 

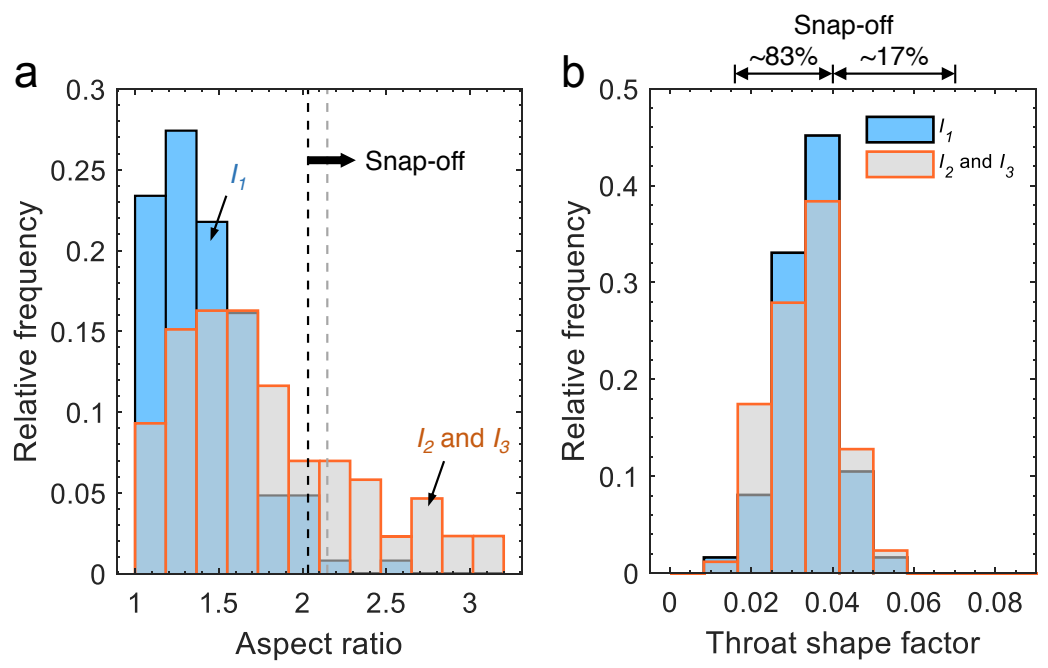

Figure 3: $I_{n}$ pore-filling, snap-off and pore-throat geometry. (a) Aspect ratio distributions for $I_{1}, I_{2}$ and $I_{3}$ events. Dashed gray and black lines show minimum (threshold) values of aspect ratios for snap-off resulting in oil trapping (for $I_{1}$ configurations) and snap-off (without trapping) between connected oil in two pores respectively. (b) Throat shape factor distribution for $I_{1}, I_{2}$ and $I_{3}$ events. Throat shape factors for snap-off events are overlain on the data along the top $x$-axis.

Table 1: Snap-off and $I_{n}$ event frequency obtained for secondary imbibition in a Ketton limestone sample.

\begin{tabular}{lcc}
\hline Event type & Frequency & Percentage \\
\hline$I_{1}$ & 124 & 67.76 \\
$I_{2}$ & 40 & 21.86 \\
$I_{3}$ & 3 & 1.64 \\
Throat snap-off with trapping & 4 & 2.19 \\
Throat snap-off without trapping & 8 & 4.37 \\
New type of pore snap-off & 4 & 2.19 \\
\hline
\end{tabular}

We first compare situations where $I_{1}$ events took place to those where throat snap-off led to oil trapping, as illustrated in Figure $1 \mathrm{~d}-\mathrm{g}$. Here we consider what we call $I_{1}$ configurations where an oilfilled pore is surrounded by throats of which only one is oil-filled. If an $I_{1}$ event occurs, both the pore and the remaining throat fill with brine and there is no trapping (see Figure 1e); if snap-off occurs in the oil-filled throat, oil is trapped in the pore (Figure $1 \mathrm{f}-\mathrm{g}$ ). Figure 3a shows the distribution of aspect ratios of the pore-throat space where various $I_{1}$ events occurred. Most of the events occur for an aspect ratio less than 1.6. On the other hand, the smallest (threshold) value of the aspect ratio for which we observe a snap-off followed by trapping is 2.15. This is in agreement with the previous subset analysis in Figure 2, and also with the theoretical value reported in the literature $(1)$. We also observed a pore-filling $I_{1}$ event which occurred for an unfavorable aspect ratio of 2.49. This pore-filling occurred immediately after a snap-off event in the adjacent pore, and may be related to inertial effects as described for event $I$ in Figure 2 and reported in a previous study (24).
Similarly, the snap-off in a throat between two pores containing connected oil (Figure $1 \mathrm{~h}-\mathrm{i}$ ) shows a good correlation with aspect ratio. A threshold value of 2.03 is found for snap-off without trapping (Figure $3 a)$. In this case, the maximum value of aspect ratio between two pores that are connected to the throat undergoing snap-off is considered.

Many $I_{2}$ pore-filling events occurred for an aspect ratio larger than 2 (Figure $3 \mathrm{a}$ ). In these events, it is likely that a snap-off occurred in one of the throats leading to an $I_{1}$ piston-like displacement in the second throat. The time resolution of tomography is not sufficient to confirm this hypothesis. This would need further investigation by conducting faster tomography or direct numerical simulations.

On the other hand, there is no clear correlation between the observed $I_{n}$ events and the throat shape factor (Figure $3 \mathrm{~b}$ ). Although a majority of snap-off events happen in throats with lower throat shape factors, this still overlaps with the distribution of throat shape factors for $I_{1}, I_{2}$ and $I_{3}$ events. This could be due to a limited voxel count in throats (due to image resolution) which does not allow for the accurate estimation of shape factors.

We have also calculated Pearson's correlations coefficient (Pearson's $r$, Eq. 1) between pore-throat geometric parameters and displacement events (snapoff, $I_{1}$ and $I_{2}$ ), as shown in Table 2. In this case, in Eq. 1, we have used $x_{i}$ as an indicator function which shows whether it is an $I_{1}$ event $($ value $=0$ ) or a snap-off (value $=1)$. The second variable $y_{i}$ is the pore-throat parameter studied (e.g., aspect ratio, pore radius, throat radius, throat shape factor and 
Table 2: Pearson's correlation coefficient, number of data points, $t$-distribution and null hypothesis probability for displacement events vs pore-throat geometry. Here, $A R$ is the aspect ratio, $R_{p}$ and $R_{t}$ are pore and throat radii respectively, $G_{t}$ is the shape factor for throats, and $Z_{p}$ is the pore coordination number. The counts $(n)$ show the total number of events identified in the analysis. In the case of $A R$ for snap-off $/ I_{1}$, a total number of 128 events were analyzed, in which 124 were $I_{1}$ and 4 snap-off with trapping, see Table 1 . In the case of $A R$ for snap-off $/ I_{2}, 40 I_{2}$ configurations were considered, in which each $I_{2}$ has two throat connections. Three of the throats were not captured clearly and were not considered in the analysis. Therefore, a total of 85 events ( 77 throats and 8 snap-off without trapping) were analyzed. Similarly, in the case of $R_{p}$ for snap-off $/ I_{1}, 128$ events were considered. In the case of $R_{p}$ for snap-off $/ I_{2}, 48$ events were analyzed, in which 40 were $I_{2}$ events and 8 snap-off without trapping (refer to Table 1).

\begin{tabular}{lcccc}
\hline Geometry and events & Pearson's $r$ & Count $(n)$ & $t$ & $p$-value \\
\hline$A R$ for snap-off $/ I_{1}$ & 0.67 & 128 & 10.02 & $9.9 \times 10^{-18}$ \\
$A R$ for snap-off $/ I_{2}$ & 0.60 & 85 & 6.85 & $1.2 \times 10^{-9}$ \\
$R_{p}$ for snap-off $/ I_{1}$ & 0.34 & 128 & 4.08 & $7.9 \times 10^{-5}$ \\
$R_{p}$ for snap-off $/ I_{2}$ & 0.45 & 48 & 3.41 & $1.4 \times 10^{-3}$ \\
$R_{t}$ for snap-off $/ I_{1}$ & -0.18 & 128 & -2.04 & 0.04 \\
$R_{t}$ for snap-off $/ I_{2}$ & -0.28 & 85 & -2.68 & $8.9 \times 10^{-3}$ \\
$G_{t}$ for snap-off $/ I_{1}$ & -0.10 & 128 & -1.12 & 0.26 \\
$G_{t}$ for snap-off $/ I_{2}$ & 0.19 & 85 & 1.77 & 0.08 \\
$Z_{p}$ for snap-off $/ I_{1}$ & 0.31 & 128 & 3.69 & $3.4 \times 10^{-4}$ \\
$Z_{p}$ for snap-off $/ I_{2}$ & -0.07 & 85 & -0.62 & 0.54 \\
\hline
\end{tabular}

Table 3: Pearson's correlation coefficient, number of data points, $t$-distribution and null hypothesis probability for event time vs pore-throat geometry. Here, $R_{p}$ and $R_{t}$ are pore and throat radii respectively, $G_{p}$ and $G_{t}$ are shape factors for pore and throat respectively, and $Z_{p}$ is the pore coordination number.

\begin{tabular}{lcccc}
\hline Event time and geometry & Pearson's $r$ & Count $(n)$ & $t$ & $p$-value \\
\hline Snap-off time and $R_{p}$ & 0.27 & 12 & 0.87 & 0.40 \\
Snap-off time and $G_{p}$ & 0.01 & 12 & 0.03 & 0.98 \\
Snap-off time and $R_{t}$ & -0.46 & 12 & -1.63 & 0.13 \\
Snap-off time and $G_{t}$ & -0.42 & 12 & -1.47 & 0.17 \\
$I_{1}$ time and $R_{p}$ & 0.30 & 124 & 3.50 & $6.5 \times 10^{-4}$ \\
$I_{1}$ time and $G_{p}$ & 0.22 & 124 & 2.52 & 0.01 \\
$I_{1}$ time and $R_{t}$ & 0.23 & 124 & 2.66 & 0.01 \\
$I_{1}$ time and $G_{t}$ & 0.17 & 124 & 1.95 & 0.05 \\
$I_{1}$ time and $Z_{p}$ & 0.14 & 124 & 1.56 & 0.12 \\
$I_{2}$ time and $R_{p}$ & 0.32 & 40 & 2.06 & 0.05 \\
$I_{2}$ time and $G_{p}$ & 0.20 & 40 & 1.27 & 0.21 \\
$I_{2}$ time and $R_{t}$ & 0.22 & 40 & 1.42 & 0.16 \\
$I_{2}$ time and $G_{t}$ & 0.20 & 40 & 1.27 & 0.21 \\
$I_{2}$ time and $Z_{p}$ & 0.15 & 40 & 0.94 & 0.35 \\
\hline
\end{tabular}

pore coordination number). A high absolute value of the Pearson's correlation coefficient indicates a strong correlation between the event type and geometric parameters. Table 2 also shows the probability of null hypothesis ( $p$-value). A high $p$-value indicates either the lack of correlation (null hypothesis) or insufficient experimental data for proving the existence of the correlation.

The analysis provided in Table 2 again shows that the aspect ratio is strongly correlated to the occurrence of snap-off or $I_{1}$ and $I_{2}$ events, with Pearson's $r$ in the range $0.60-0.67$ and low $p$-values. This dependence on aspect ratio is not explicitly implemented in many pore network models to capture the competition between snap-off and pore filling $(41,42)$, and can be used to improve the accuracy of these models.

Similarly, pore radius $\left(R_{p}\right)$ for snap-off $/ I_{1}$ and snapoff $/ I_{2}$ shows a good correlation with low $p$-values, indicating that pore filling is suppressed when the pore involved is large; however, throat radius $\left(R_{t}\right)$ shows a poor correlation with high $p$-values. This shows that just having a small throat does not necessarily favor snap-off over pore-filling: the key criterion is the throat radius in relation to the pore, captured by the aspect ratio. On the other hand, pore coordination number for snap-off $/ I_{1}$ shows a good correlation with a low $p$-value. Snap-off is more likely in the remaining throat in pores with a larger coordination number: here it is because the more connected pores tend to be larger with a higher aspect ratio. The coordination number 
analysis for snap-off $/ I_{2}$ events shows a poor correlation, which could be possibly due to misidentification (due to limited time resolution) of $I_{2}$ events compared to snap-off followed by an $I_{1}$ event.

The throat shape factor shows weaker correlations with higher probability of null hypothesis (Table 2 ), which confirms the findings reported in Figure 3b. This indicates that the implementation of the dependence of displacement events on the throat shape factor in pore-network models could produce biased results, and must be carefully evaluated.

The time at which these events happen gives an indication of the order in which an event is preferred and the resulting fluid distribution. Table 3 shows the correlation of the pore-throat geometry and the time when events took place. In this case, we have used the event time as $x_{i}$ variable and pore-throat parameters (e.g., pore radius) as $y_{i}$ variable in Eq. 1. For $I_{1}$ and $I_{2}$ events, only pore radius shows a good correlation and has low $p$-values. Other parameters, such as shape factor and coordination number show a reasonable correlation, however with high $p$-values.

\section{Pore radius of displacement events as a function of their time of occurrence}

Figure 4 shows pore radii for various events as a function of time. Although larger pores appear to be filled at later times, which is expected as the capillary pressure decreases, we still observe many small pores experiencing $I_{1}$ events at later times. This could be due to transition of configurations from $I_{2}$ to $I_{1}$ with fluid displacement. With continuous brine injection, it is possible that one of the throats in an $I_{2}$ configuration is filled due to either a snap-off in the throat or $I_{1}$ displacement in the adjacent pore. This leaves the pore in question with an $I_{1}$ configuration at late times. As described in the schematic in Figure $1 \mathrm{a}-\mathrm{b}$, an $I_{1}$ event is more favorable than the original $I_{2}$ event for the same pore size and can happen at a higher capillary pressure. For this reason, small pores changing from an $I_{2}$ to an $I_{1}$ configuration at later stages of brine injection are potentially in an unstable configuration and will be rapidly filled with brine.

Similarly, invasion of an $I_{2}$ configuration for smaller pore radii at later times was observed. In $I_{2}$ configurations, snap-off in one of the throats could have preceded the actual pore filling event, making the latter an $I_{1}$ rather than an $I_{2}$ invasion. Such events may have been misidentified in the images due to limited time resolution. This hypothesis is supported by the aspect ratio distribution (Figure 3a)

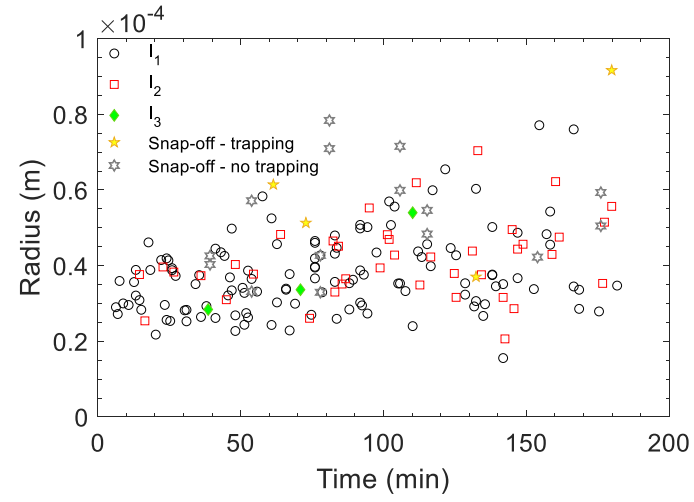

Figure 4: Pore radius for various snap-off and $I_{n}$ events as a function of time.

in which we observe many $I_{2}$ events for aspect ratios more than 2, which tend to be favorable for snap-off. Therefore, the pore radii cross-plot with time for $I_{2}$ events (Figure 4) could be biased, and this is likely to affect its correlation with time. An example of such an event transition can be seen in Figure $2 \mathrm{~g}$-i, in which snap-off event $F$ changes the fluid configuration in the adjacent pore (marked by cyan circle in Figure 2i) from $I_{3}$ (before snap-off) to $I_{2}$ (after snap-off), thereby facilitating the displacement of oil through event $G$ and $H$ after snap-off. Note that the radius of this particular pore (marked by cyan circle in Figure 2i) is smaller compared to two other pores that were invaded through porefilling events in earlier times (events $D$ and $E$ ), and could be expected to fill first. However, this did not take place due to the $I_{3}$ configuration in this pore prior to snap-off, which impedes pore-filling. With its transition from $I_{3}$ to $I_{2}$, a pore-filling becomes more favorable.

\section{Unexpected $I_{2}$ pore-filling event}

We have observed an $I_{2}$ pore-filling event with unfavorable aspect ratios. Figure $5 \mathrm{a}$ shows an $I_{2}$ fluid configuration where two throats connected to a pore are occupied by oil. In the next time step, oil is displaced to the adjacent pores through both throats (Figure 5b). The aspect ratios involved in the displacement events $A$ and $B$ are 3.3 and 4.4 respectively, which are favorable for snap-off and oil trapping; however, the snap-off does not occur in any of the throats, rather the oil migrates to the adjacent pores without being trapped. It is also possible that a snap-off event occurred in one of the throats forming an $I_{1}$ configuration in the second throat in which the displacement could occur due to inertial effects after a snap-off in the neighbouring throat. To explain this type of displacement event, further research is needed. 
a

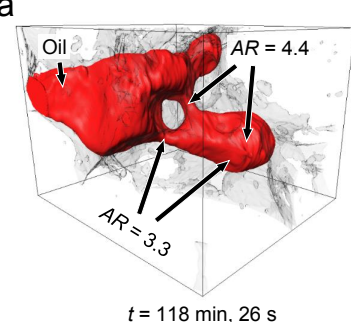

b

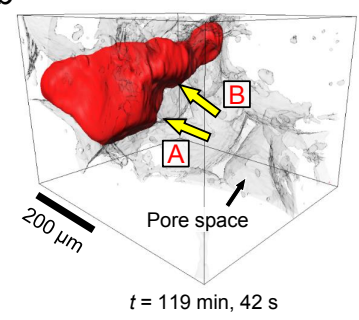

Figure 5: Unexpected $I_{2}$ pore-filling event. (a) Oil in $I_{2}$ configuration at $t=118 \mathrm{~min}, 26 \mathrm{~s}$. (b) The oil-brine interface is displaced to adjacent pores in the next time step at $t=$ $119 \mathrm{~min}, 42 \mathrm{~s}$. Here, $A R$ is the aspect ratio.

\section{New type of pore snap-off leading to oil trapping}

We have found a new type of displacement event leading to the formation of sub-singlet ganglia (occupying less than a pore body) in the pore space. Figure 6 shows the formation of such a ganglion, in which the oil in an $I_{1}$ fluid configuration (Figure 6a) is displaced to the adjacent pore (through throat 3 ) in the next time step (Figure 6b). The aspect ratio of the pore-throat configuration is 2.7 , therefore a traditional snap-off in throat 3 leading to trapping in the middle of the pore space was expected. However, neither a complete pore-filling nor a snap-off (due to brine layer swelling) occurred in this case, rather the oil-brine interface jumped to the adjacent pore leaving a small fraction of oil which appears to be in a pore corner close to throat 2 . The oil appears to be stuck to the pore wall crevice, and does not occupy the larger space in the center of the pore as in the case of trapping after a traditional snap-off (18).

This type of snap-off and trapping has similarities to the fragmentation of a non-wetting phase (oil) observed during desaturation at fast flow rates (43); however, it cannot be related to the same mechanisms as the flow rate in our study is slower by four orders of magnitude. It may be argued that the interfacial velocity during pore-filling in a slow capillary-dominated flow might be extremely high to result such a fragmentation; however, we hypothesize that the formation of such ganglia is likely to be due to interface pinning at rough edges and corners of the pore space.

The interface pinning at solid surfaces can be observed in Figure 6c, which results in an advancing contact angle more than $90^{\circ}$ during displacement. Using direct numerical simulations (44), we show that this pinning holds the interface at the threephase contact points on the solid surfaces; however, with the increasing water pressure (or decreasing

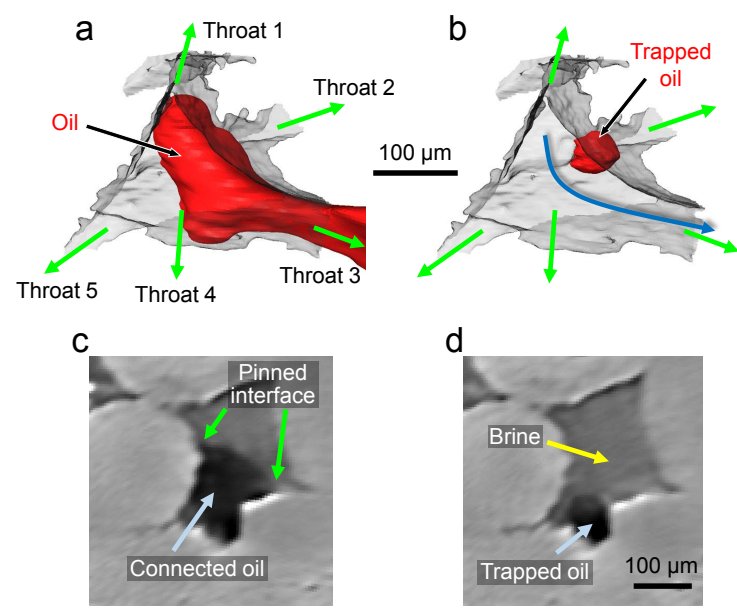

Figure 6: New type of pore snap-off leading to the trapping of a non-wetting phase (oil). (a) Oil in $I_{1}$ configuration at $t=128 \mathrm{~min}, 34 \mathrm{~s}$. (b) In the next time step at $t=129$ min, $50 \mathrm{~s}$, the oil-brine interface jumps to the adjacent pore, leaving behind a small fraction of oil in the pore space. (cd) A two-dimensional slice of the original gray-scale image showing (c) the pinned interface before trapping (at $t=128$ min, $34 \mathrm{~s}$ ), and (d) the trapped non-wetting (oil) phase (at $t=129 \mathrm{~min}, 50 \mathrm{~s})$.

capillary pressure), the water continues to displace the oil in the centre of the pore body. This eventually empties the pore centre, leaving the oil stuck at the pore walls. Once the oil is disconnected, the interfaces rearrange to attain an equilibrium pressure condition (Figure 6d).

We have found four such sub-singlet ganglia formed in single pore bodies in our time-series data. The pore-throat aspect ratio in these events is the range of 1.6 to 2.7 , which indicates that this type of pore snap-off is independent of aspect ratio. Although these events result in trapping of small volumes of oil which may not be significant for oil recovery applications, it would be crucial for designing effective remediation techniques for non-aqueous phase liquid contaminated soils.

\section{$I_{2}$ pore-filling, bypassing and trapping}

Apart from snap-off, the second type of trapping is due to pore-filling and bypassing. This is illustrated in Figure 7 . Here, an $I_{2}$ pore-filling occurs in either Pore 1 or Pore 2, leading to oil bypassing and trapping in two pore bodies. Aspect ratios of the pore and throats involved in this event are small (in the range 1.1 to 1.9 , considering both throats connected to Pore 1 and Pore 2), therefore a snap-off is not favorable. This type of bypassing and trapping, which can lead to the formation of large ganglia occupying several pore bodies, has been widely investigated, especially in a pore doublet geometry 
in micromodels (45); however, this is the first time that it has been demonstrated in three dimensions using a time-resolved study.

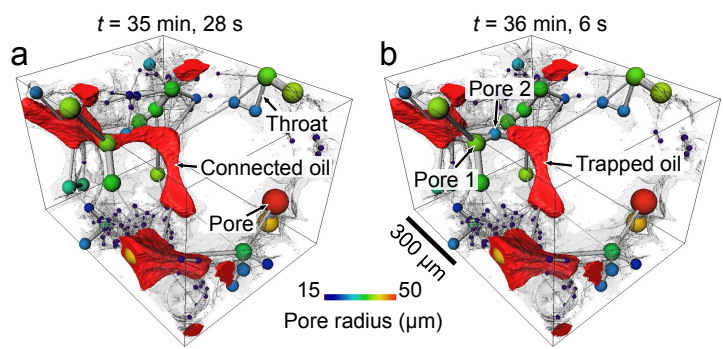

Figure 7: $I_{2}$ pore-filling, bypassing and trapping. (a-b) Oil configuration in two consecutive tomographic time steps: (a) connected oil, and (b) trapped oil. Oil in the pore space is superimposed on pores (colored spheres) and throats (gray tubes).

\section{Conclusions}

We have used fast synchrotron X-ray microtomography to investigate various displacement events such as $I_{n}$ pore-filling, snap-off, bypassing and trapping during secondary imbibition in a Ketton limestone sample. By extracting a pore-throat network and superimposing it on the oil phase in consecutive time-series images, we have analyzed conditions that are favorable for each type of event. The Pearson's correlation coefficient and probability of null hypothesis showed that the aspect ratio has a strong influence on whether a snap-off or a pore-filling event occurred. Aspect ratios less than 2.03 were found to be favorable for pore-filling, except for two pore-filling events that occurred for values of 2.1 and 2.49. This unfavorable pore-filling may be related to inertial effects, especially after a snap-off event in the adjacent throat; however, further research is needed to support this hypothesis. On the other hand, throat shape factors were weakly correlated to snap-off and $I_{n}$ events. This may have been influenced by the limited number of voxels in a throat, which did not allow us to resolve its shape accurately. Moreover, the pore radius in $I_{1}$ and $I_{2}$ events was found to be positively correlated to event time or, equivalently, to a decreasing capillary pressure with time.

We have also observed a new type of pore snap-off leading to oil trapping in a fraction of a pore body, which is related to interface pinning at rough pore surfaces. Finally, we have also identified an unexpected $I_{2}$ pore-filling event for high aspect ratios that were favorable for snap-off, however a pistonlike displacement occurred through both throats. Future work can focus on faster synchrotron imaging or the visualization of flow in three-dimensional printed micromodels along with direct numerical simulations, to investigate these processes in detail.

Overall, the analysis and correlations provided in this study are expected to improve our general understanding of the physics of fluid flow in water-wet porous media, and consequently of pore-scale models of fluid displacement processes. Further experiments and similar analysis on porous media with altered wettability, with different initial conditions, or on other types of porous media, e.g., sand packs, sandstones and more complex carbonate rocks, can provide the generality of our approach.

Acknowledgements. We gratefully acknowledge funding from the Qatar Carbonates and Carbon Storage Research Centre (QCCSRC), provided jointly by Qatar Petroleum, Shell, and Qatar Science and Technology Park. We thank Hannah Menke, Matthew Andrew, Branko Bijeljic, Tarik Saif, Norman Nicholls, Joan Vila-Comamala and Christoph Rau for help in imaging. We thank Diamond Light Source for access to beamline I13 (MT11587). T.B. is a postdoctoral fellow of the Research Foundation-Flanders (FWO) and acknowledges its support under grant 12X0919N. A.Q.R thanks TOTAL and Wintershall Dea for their financial support. M.S. acknowledges the support of the Natural Environment Research Council via grant NE/N016173/1.

Author contributions. K.S. and M.J.B. designed research. K.S., T.B., A.Q.R., M.S., and M.J.B conducted data analysis and interpreted the results. K.S. wrote the manuscript with input from all other authors.

Competing interests. The authors declare that they have no competing interests.

\section{References}

(1) Blunt, M. J., Multiphase Flow in Permeable Media: A Pore-Scale Perspective; Cambridge University Press: 2017.

(2) Zhao, B., MacMinn, C. W., and Juanes, R., (2016). Wettability control on multiphase flow in patterned microfluidics. Proceedings of the $\mathrm{Na}$ tional Academy of Sciences 113, 10251-10256.

(3) Singh, K., Jung, M., Brinkmann, M., and Seemann, R., (2019). Capillary-dominated fluid displacement in porous media. Annual Review of Fluid Mechanics 51, 429-449.

(4) Lenormand, R., Zarcone, C., and Sarr, A., (1983). Mechanisms of the displacement of one fluid by another in a network of capillary ducts. Journal of Fluid Mechanics 135, 337-353.

(5) Pavuluri, S., Maes, J., Yang, J., Regaieg, M., Moncorgé, A., and Doster, F., (2019). Towards pore network modelling of spontaneous imbibition: Contact angle dependent invasion patterns and the occurrence of dynamic capillary barriers. Computational Geosciences. 
(6) Zacharoudiou, I., Chapman, E. M., Boek, E. S., and Crawshaw, J. P., (2017). Pore-filling events in single junction micro-models with corresponding lattice Boltzmann simulations. Journal of Fluid Mechanics 824, 550-573.

(7) Holtzman, R., and Segre, E., (2015). Wettability stabilizes fluid invasion into porous media via nonlocal, cooperative pore filling. Phys. Rev. Lett. 115, 164501.

(8) Hu, R., Wan, J., Yang, Z., Chen, Y.-F., and Tokunaga, T., (2018). Wettability and flow rate impacts on immiscible displacement: A theoretical model. Geophysical Research Letters 45, 3077-3086.

(9) Haines, W. B., (1930). Studies in the physical properties of soil. V. The hysteresis effect in capillary properties, and the modes of moisture distribution associated therewith. The Journal of Agricultural Science 20, 97-116.

(10) Berg, S., Ott, H., Klapp, S. A., Schwing, A., Neiteler, R., Brussee, N., Makurat, A., Leu, L., Enzmann, F., Schwarz, J.-O., Kersten, M., Irvine, S., and Stampanoni, M., (2013). Realtime 3D imaging of Haines jumps in porous media flow. Proceedings of the National Academy of Sciences 110, 3755-3759.

(11) Bultreys, T., Boone, M., Boone, M., De Schryver, T., Masschaele, B., Van Loo, D., Van Hoorebeke, L., and Cnudde, V., (2015). Realtime visualization of Haines jumps in sandstone with laboratory-based microcomputed tomography. Water Resources Research 51, 8668-8676.

(12) Singh, K., Drainage in carbonate rocks: high-resolution time-resolved synchrotron X-ray micro-tomography., Figshare, https : / / doi . org/10.6084/m9.figshare.7082885.v1, 2018.

(13) Andrew, M., Menke, H., Blunt, M. J., and Bijeljic, B., (2015). The imaging of dynamic multiphase fluid flow using synchrotron-based X-ray microtomography at reservoir conditions. Transport in Porous Media 110, 1-24.

(14) Yu, L., and Wardlaw, N. C., (1986). Mechanisms of nonwetting phase trapping during imbibition at slow rates. Journal of Colloid and Interface Science 109, 473-486.

(15) Pickell, J. J., Swanson, B. F., and Hickman, W. B., (1966). Capillary pressure data in the study of pore structure and fluid distribution. Society of Petroleum Engineers Journal 6, 5561.

(16) Lenormand, R., and Zarcone, C., (1984). Role of roughness and edges during imbibition in square capillaries. SPE 13264, proceedings of the $59^{\text {th }}$ SPE Annual Technical Conference and Exhibition, Houston, Texas, 16-19 September.
(17) Ruspini, L., Farokhpoor, R., and Øren, P., (2017). Pore-scale modeling of capillary trapping in water-wet porous media: A new cooperative pore-body filling model. Advances in Water Resources 108, 1-14.

(18) Singh, K., Menke, H., Andrew, M., Lin, Q., Rau, C., Blunt, M. J., and Bijeljic, B., (2017). Dynamics of snap-off and pore-filling events during twophase fluid flow in permeable media. Scientific Reports 7, 5192.

(19) Nguyen, V. H., Sheppard, A. P., Knackstedt, M. A., and Pinczewski, W. V., (2006). The effect of displacement rate on imbibition relative permeability and residual saturation. Journal of Petroleum Science and Engineering 52, Reservoir Wettability, 54-70.

(20) Yu, L., and Wardlaw, N. C., (1986). The influence of wettability and critical pore-throat size ratio on snap - off. Journal of Colloid and Interface Science 109, 461-472.

(21) Chatzis, I., Morrow, N. R., and T., L. H., (1983). Magnitude and detailed structure of residual oil saturation. Society of Petroleum Engineers Journal 23, 311-326.

(22) Patzek, T., and Silin, D., (2001). Shape factor and hydraulic conductance in noncircular capillaries: I. One-phase creeping flow. Journal of Colloid and Interface Science 236, 295-304.

(23) Singh, K., Scholl, H., Brinkmann, M., Michiel, M. D., Scheel, M., Herminghaus, S., and Seemann, R., (2017). The role of local instabilities in fluid invasion into permeable media. Scientific Reports 7, 444.

(24) Rücker, M., Berg, S., Armstrong, R. T., Georgiadis, A., Ott, H., Schwing, A., Neiteler, R., Brussee, N., Makurat, A., Leu, L., Wolf, M., Khan, F., Enzmann, F., and Kersten, M., (2015). From connected pathway flow to ganglion dynamics. Geophysical Research Letters 42, 38883894 .

(25) Armstrong, R. T., Georgiadis, A., Ott, H., Klemin, D., and Berg, S., (2014). Critical capillary number: Desaturation studied with fast Xray computed microtomography. Geophysical Research Letters 41, 55-60.

(26) Schlüter, S., Berg, S., Rücker, M., Armstrong, R. T., Vogel, H.-J., Hilfer, R., and Wildenschild, D., (2016). Pore-scale displacement mechanisms as a source of hysteresis for two-phase flow in porous media. Water Resources Research 52, 2194-2205.

(27) Schlüter, S., Berg, S., Li, T., Vogel, H.-J., and Wildenschild, D., (2017). Time scales of relaxation dynamics during transient conditions in two-phase flow. Water Resources Research 53, 4709-4724. 
(28) Berg, S., Armstrong, R. T., Georgiadis, A., Ott, H., Schwing, A., Neiteler, R., Brussee, N., Makurat, A., Rucker, M., Leu, L., Wolf, M., Khan, F., Enzmann, F., and Kersten, M., (2015). Onset of oil mobilization and nonwetting-Phase clustersize distribution. Petrophysics 56, 15-22.

(29) Herring, A. L., Robins, V., and Sheppard, A. P., (2019). Topological persistence for relating microstructure and capillary fluid trapping in sandstones. Water Resources Research 55, 555-573.

(30) Andersson, L., Herring, A., Schlüter, S., and Wildenschild, D., (2018). Defining a novel porebody to pore-throat "Morphological Aspect Ratio" that scales with residual non-wetting phase capillary trapping in porous media. Advances in Water Resources 122, 251-262.

(31) Tanino, Y., and Blunt, M. J., (2012). Capillary trapping in sandstones and carbonates: Dependence on pore structure. Water Resources Research 48 .

(32) Raeini, A. Q., Bijeljic, B., and Blunt, M. J., (2017). Generalized network modeling: Network extraction as a coarse-scale discretization of the void space of porous media. Phys. Rev. E 96, 013312 .

(33) Raeini, A. Q., Yang, J., Bondino, I., Bultreys, T., Blunt, M. J., and Bijeljic, B., (2019). Validating the generalized pore network model using micro-CT images of two-phase flow. Transport in Porous Media.

(34) Bulreys, T., Singh, K., Raeini, A. Q., Ruspini, L. C., Øren, P. E., Berg, S., Rücker, M., Bijeljic, B., and Blunt, M. J., (2019). Verifying simple models with complex emergent behaviour: fluid invasion into porous materials (in preparation).

(35) Andrew, M., Bijeljic, B., and Blunt, M. J., (2014). Pore-scale imaging of trapped supercritical carbon dioxide in sandstones and carbonates. International Journal of Greenhouse Gas Control 22, 1-14.

(36) Singh, K., Menke, H., Andrew, M., Rau, C., Bijeljic, B., and Blunt, M. J., (2018). Time-resolved synchrotron X-ray micro-tomography datasets of drainage and imbibition in carbonate rocks. Scientific Data 5, 180265.

(37) Buades, A., Coll, B., and Morel, J., (2008). Nonlocal image and Movie denoising. International Journal of Computer Vision 76, 123-139.

(38) Singh, K., Curvature mapping during porefilling and snap-off event in water-wet carbonate rock., Figshare, https://doi.org/10.6084/m9. figshare.4235381.v1, 2017.

(39) Singh, K., Pore-filling and snap-off events superimposed on a pore-throat network extracted from the segmented pore space., Figshare, https:// doi . org/10.6084/m9 . figshare . 9034367 . v1, 2019 .
(40) Bultreys, T., Lin, Q., Gao, Y., Raeini, A. Q., AlRatrout, A., Bijeljic, B., and Blunt, M. J., (2018). Validation of model predictions of porescale fluid distributions during two-phase flow. Phys. Rev. E 97, 053104.

(41) Valvatne, P. H., and Blunt, M. J., (2004). Predictive pore-scale modeling of two-phase flow in mixed wet media. Water Resources Research 40.

(42) Øren, P. E., and Blunt, M. J., (1998). Extending predictive capabilities to network models. SPE Journal 3, 324-336.

(43) Pak, T., Butler, I. B., Geiger, S., van Dijke, M. I. J., and Sorbie, K. S., (2015). Droplet fragmentation: 3D imaging of a previously unidentified pore-scale process during multiphase flow in porous media. Proceedings of the National Academy of Sciences 112, 1947-1952.

(44) Shams, M., Singh, K., Bijeljic, B., and Blunt, M. J., (2019). Direct numerical simulation of pore-scale trapping events during capillarydominated two-phase flow in porous media (in preparation).

(45) Chatzis, I., and Dullien, F., (1983). Dynamic immiscible displacement mechanisms in pore doublets: Theory versus experiment. Journal of Colloid and Interface Science 91, 199-222. 